\title{
Seasonal Nutrient Cycling and Enrichment of Nutrient-Related Soil Microbes Aid in the Adaptation of Ramie (Boehmeria nivea L.) to Nutrient-Deficient Conditions
}

\author{
Shenglan $W u^{1,2}$, Shuai $X_{u e^{3}}$, Yasir lqbal ${ }^{3}$, Hucheng Xing ${ }^{1}$ and Yucheng Jie ${ }^{1 *}$ \\ ${ }^{1}$ College of Agronomy, Hunan Agricultural University, Changsha, China, ${ }^{2}$ Orient Science \& Technology College of Hunan \\ Agricultural University, Changsha, China, ${ }^{3}$ College of Bioscience \& Biotechnology, Hunan Agricultural University, Changsha, \\ China
}

The breeding for varieties tolerant of adverse growing conditions is critical for sustainable agriculture, especially for ramie (Boehmeria nivea L.). However, a lack of information on the tolerance of ramie to nutrient-deficient conditions has hindered efforts to breed ramie

OPEN ACCESS

Edited by:

Victoria Fernandez,

Polytechnic University of Madrid,

Spain

Reviewed by:

Lijun Liu,

Huazhong Agricultural University,

China

Andriele Wairich,

Embrapa Uva e Vinho, Brazil

*Correspondence:

Yucheng Jie

ibfcjyc@vip.sina.com

Specialty section:

This article was submitted to

Plant Nutrition,

a section of the journal

Frontiers in Plant Science

Received: 23 December 2020

Accepted: 26 February 2021

Published: 31 March 2021

Citation:

Wu S, Xue S, lqbal Y, Xing H and Jie Y (2021) Seasonal Nutrient Cycling and Enrichment of Nutrient-Related Soil Microbes Aid in the Adaptation of Ramie (Boehmeria nivea L.)

to Nutrient-Deficient Conditions.

Front. Plant Sci. 12:644904.

doi: 10.3389/fp/s.2021.644904 varieties tolerant of such conditions. The main objective of this study was to explore the tolerance strategies of ramie plants under poor soil conditions using long-term (89 years) field trials. Genotypes of Duobeiti 1 and Xiangzhu XB were highly tolerant of poor soil conditions. The contributions of seasonal nutrient cycling and rhizobacteria to the ability of ramie to tolerate poor soil were tested. Nitrogen and phosphorus retranslocation to the root at the end of the growing season helped ramie adapt to poor soil conditions. The contribution of the microbial community was analyzed using high-throughput Illumina MiSeq sequencing technology. The enrichment of beneficial bacteria (mainly Bradyrhizobium, Gaiella, and norank_o_Gaiellales) and the reduction of harmful fungi (mainly Cladosporium and Aspergillus) also contributed to the ability of ramie to tolerate poor soils. The results of this study provide new insight into the ability of ramie to tolerate adverse conditions and aid future efforts to breed and cultivate ramie tolerant of adverse conditions.

Keywords: fibrous crop, nutrient retranslocation, microbial communities, tolerance strategy, infertile soil

\section{INTRODUCTION}

Ramie (Boehmeria nivea L.), commonly known as China grass, is a hardy perennial herbaceous plant (Urticaceae: tribe Boehmerieae). Cells in its stem phloem are long, with a maximum length of $60 \mathrm{~cm}$, and are made up of nearly pure cellulose (ca. 96\%) (Satya et al., 2019). These long bast cells have traditionally been used as natural fibers to produce a variety of products, such as cloth, finishing net, and upholstery fabrics. With the increase in the demand for natural fibers, ramie has become an important industrial crop; it is mainly cultivated in Asian countries (Rehman et al., 2019). China contributes to more than $96 \%$ of the world's ramie production (FAOSTAT) ${ }^{1}$. However,

\footnotetext{
${ }^{1}$ http://faostat.fao.org
} 
there has been a considerable decline in the global area under ramie cultivation: from 143,952 ha in 2007 to 52,884 ha in 2018 . The decline has been primarily caused by the replacement of ramie with other crops (mainly Oryza sativa L.) in China.

The low profitability of ramie production, which stems from the increasing input costs but low and stable fiber price, is the main factor contributing to the reduction in the area under ramie cultivation in China. Multiple (usually two to three) midseason harvests result in the removal of macroelements (i.e., uptake by the plants) such as nitrogen $(\mathrm{N})$, phosphorus $(\mathrm{P})$, and potassium (K) from the soil (Liu et al., 2013). Consequently, heavy fertilizer application is essential for achieving stable yields and the production of high-quality fiber (Tatar et al., 2010). Ramie cultivation could be improved if fertilizer inputs could be reduced. Although optimizing cultivation techniques (e.g., fertilization or harvesting strategies) could reduce fertilizer input (Liu et al., 2012; Zeng et al., 2013), the breeding for varieties that can use nutrients efficiently and grow on poor soils will provide a more efficient and long-term solution. Additionally, given that ramie shows strong heavy metal tolerance and has a variety of nonfood uses (Rosariastuti et al., 2018; Lan et al., 2020), ramie could be grown in heavy metal-contaminated soil (Neilson and Rajakaruna, 2014; Zheng J. et al., 2019). Heavy metal-contaminated soils (mainly in mining areas) are generally characterized by poor fertility (Mensah, 2015; Lwin et al., 2018). Therefore, expanding ramie cultivation to these areas requires breeding varieties that can tolerate soils with poor fertility.

There has been a little effort to develop new ramie varieties with the potential to grow in poor soil. A lack of information on how ramie plants tolerate nutrient-deficient soil conditions (i.e., their tolerance strategy) has hindered breeding efforts. The aim of this study was to investigate the strategies by which ramie plants cope with poor soil conditions. In perennial plants, nutrient retranslocation (or seasonal nutrient cycling) plays an important role in maintaining their normal growth when nutrients are limited (Fife et al., 2008; Moreira and Fageria, 2009; Erley et al., 2010), especially for plants with extensive underground root systems, such as miscanthus (Miscanthus spp.) and giant reed (Arundo donax L.) (Nasso et al., 2011, 2013; Ruf et al., 2017). Ramie is a perennial herb characterized by a well-developed underground root system composed of rhizomes, radish roots, and fine roots. One possible tolerance strategy is via the seasonal cycling of macronutrients, which could improve the adaptation of ramie to nutrient-deficient conditions. To test for this strategy, relationships between the seasonal rates of change in $\mathrm{N}, \mathrm{P}$, and $\mathrm{K}$ and ramie's poor soil tolerance index were characterized. In previous studies, rhizobacteria were found to play a major role in the tolerance of plants to poor soil by mediating the process of $\mathrm{N}$ cycling and plant nutrient acquisition (Liu and Ludewig, 2019). The soil microbial community might also contribute to the tolerance of ramie to poor soil. To test for this strategy, the relationship between the structure of the soil microbial community and ramie's poor soil tolerance index was assessed. The above two strategies were tested using different germplasm materials in two long-term field trials.

\section{MATERIALS AND METHODS}

\section{Site Conditions and Experimental Design}

This study was conducted by establishing ramie stands at the experimental stations of Changsha (ECS, $28^{\circ} 11^{\prime} 10^{\prime \prime} \mathrm{N}, 113^{\circ} 4^{\prime} 5^{\prime \prime} \mathrm{E}$, $58 \mathrm{~m}$ a.s.l.) and Huarong (EHR, $29^{\circ} 32^{\prime} 46^{\prime \prime} \mathrm{N}, 112^{\circ} 39^{\prime} 57^{\prime \prime} \mathrm{E}$, $73 \mathrm{~m}$ a.s.1.). The ECS site is characterized by red clay soil (collected in November 2017) with poor fertility [total nitrogen (TN) $0.69 \mathrm{~g} / \mathrm{kg}$, available phosphorus (AP) $9.62 \mathrm{mg} / \mathrm{kg}$, and exchangeable potassium (EP) $56.53 \mathrm{mg} / \mathrm{kg}$ ], whereas the EHR site is a wasteland with a poor sandy red soil (TN $0.66 \mathrm{~g} / \mathrm{kg}$, AP $7.61 \mathrm{mg} / \mathrm{kg}$, and EP $59.54 \mathrm{mg} / \mathrm{kg}$; collected in November 2018). Both sites feature a humid subtropical monsoon climate with a long-term annual average precipitation of 1,420 and $1,062 \mathrm{~mm}$ and air temperature of 17.1 and $16.7^{\circ} \mathrm{C}$ for ECS and EHR, respectively.

Field trials at both sites were conducted in spring 2010. Plant materials used for each trial are shown in Table 1. Experiments were conducted in a randomized block design (block size of $4 \times 2.5 \mathrm{~m}$ ) with three replicates. All materials were planted using $300 \mathrm{~g}$ of rhizome (collected from the germplasms garden of our team at Changsha) at a density of four plants $/ \mathrm{m}^{2}$ (i.e., $0.5 \times 0.5 \mathrm{~m}$ spacing). Soil tillage and harrowing were conducted before planting. After planting, only two harvests per year (July and December) were carried out. No other management practices (e.g., irrigation, weeding, and pest control) were used.

\section{Sampling Strategy and Data Collection Evaluation of Ramie Field Performance}

For the ECS trial, the field performance of established plants was measured in November of both the 2010 (the establishment year) and 2018 growing seasons. Field performance was also assessed in the middle of July 2018 near the mid-season harvest. Similarly, morphological features of the EHR plants were measured in mid-July and the end of November 2019. The morphological characteristics included plant height, tiller number, and aboveground and belowground biomass. The heights of 10 randomized selected plants within each plot were recorded. At each sampling event, an area of $0.5 \mathrm{~m}^{2}$ in each plot was randomly chosen using a $0.5 \times 1-\mathrm{m}$ quadrat so that the distance between the quadrat boundary and plot boundary was no less than $30 \mathrm{~cm}$. The aboveground biomass within the quadrat

TABLE 1 | Information of the ramie materials used in the two field trials.

\begin{tabular}{lcll}
\hline Material name & Abbreviation & Material type & Used in trial \\
\hline Xiangzhu X1 & XZ-X1 & Genotype & ECS trial \\
Xiangzhu X2 & XZ-X2 & Genotype & ECS trial and EHR trial \\
Xiangzhu X3 & XZ-X3 & Genotype & ECS trial \\
Xiangzhu XB & XZ-XB & Genotype & ECS trial and EHR trial \\
Xiangzhu 3 & XZ-3 & Variety & ECS trial and EHR trial \\
Duobeiti 1 & D-1 & Genotype & ECS trial \\
Zhongzhu 1 & ZZ-1 & Variety & EHR trial \\
\hline
\end{tabular}

ECS and EHR represent the trials established at the experimental stations of Changsha and Huarong, respectively. 
was then harvested. In addition, the belowground root system, including the rhizome, radish root, and fine root (hereafter referred to collectively as "root") was excavated (to a depth of $40 \mathrm{~cm}$ ), washed, and collected. All of the biomass collected was dried to a constant weight of $60^{\circ} \mathrm{C}$ and then weighed to collect data on biomass yield.

\section{Evaluation of the Plant Nutrient Concentration}

For the ECS field trial, plant nutrient concentration was analyzed based on samples that were collected monthly from April to December 2018. In the middle of each sampling month, aboveground and belowground biomass within $0.5 \mathrm{~m}^{2}$ was harvested as described above. The aboveground plants were separated into stem and leaves. All subsamples were dried to a constant weight at $60^{\circ} \mathrm{C}$. Afterward, each dried sample was weighed and milled to powder (100 mesh) for subsequent nutrient concentration analysis. The nitrogen concentration was determined by the Kjeldahl method using a K9840 distillator/titrator (Haineng, China). The phosphorus concentration was determined with a UV-2700i spectrophotometer (Shimadzu, Japan) at $700 \mathrm{~nm}$ using the molybdenum blue method. The K concentrations were analyzed with the flame photometry method using an FP6450 photometer (SHjingmi, China). These analysis methods are described in detail in the work of Bao (2000). Samples for all nutrient analyses were subjected to $\mathrm{H}_{2} \mathrm{SO}_{4}-\mathrm{H}_{2} \mathrm{O}_{2}$ digestion. Each sample was analyzed three times, and the mean value was used in the analyses.

\section{Soil Microbial Composition Analysis}

In this study, soil microbial composition analysis was conducted using the soil collected from the EHR trial in October 2019. High-throughput Illumina MiSeq sequencing technology was used to analyze the structure of soil microbial (including fungi and bacteria) communities. Five plants from each plot were randomly selected according to the "S" shape principle. Their rhizosphere soils were then collected to prepare a composite sample by removing and mixing the soil from rhizomes and roots. Composite soil was mixed, sieved $(2 \mathrm{~mm})$, reduced to $50 \mathrm{~g}$, and finally stored in liquid nitrogen until high-throughput sequencing analysis.

High-throughput sequencing analysis generally involves three steps: DNA extraction, PCR amplification, and Illumina MiSeq sequencing. DNA of the total microbial community was first isolated from the aforementioned samples using the E.Z.N.A. ${ }^{\circledR}$ soil DNA Kit (Omega Bio-tek, Norcross, GA, United States) as per the manufacturer's instructions. The DNA extract was then tested on $1 \%$ agarose gel, and DNA concentration and purity were determined with a NanoDrop 2000 UV-Vis spectrophotometer (Thermo Scientific, Wilmington, MA, United States). The tested DNA samples were amplified using an ABI GeneAmp ${ }^{\circledR} 9700$ PCR thermocycler (ABI, CA, United States). The primer pairs of $338 \mathrm{~F} / 806 \mathrm{R}$ were used to amplify the hypervariable region V3-V4 of the bacterial 16S rRNA gene and ITS1F/ITS2R to the internal transcribed spacer (ITS) region of fungal rRNA genes. Both PCR reactions were performed as follows: initial denaturation at $95^{\circ} \mathrm{C}$ for $3 \mathrm{~min} ; 27$ cycles of denaturation at $95^{\circ} \mathrm{C}$ for $30 \mathrm{~s}$, annealing at $55^{\circ} \mathrm{C}$ for $30 \mathrm{~s}$, and extension at $72^{\circ} \mathrm{C}$ for $45 \mathrm{~s}$; and a final extension at $72^{\circ} \mathrm{C}$ for $10 \mathrm{~min}$. The TransGen AP22102 (TransStart Fastpfu DNA Polymerase) mixture and TaKaRa rTaq DNA Polymerase mixture were used for the PCR of the bacterial 16S rRNA gene and fungal rRNA genes, respectively; details of the procedure are provided in Zhang et al. (2019). The PCR products were extracted from a $2 \%$ agarose gel and purified using the AxyPrep DNA Gel Extraction Kit (Axygen Biosciences, Union City, CA, United States) as per the manufacturer's instructions and quantified using a Quantus ${ }^{\mathrm{TM}}$ Fluorometer (Promega, United States). Purified amplicons were pooled in equimolar amounts and paired-end sequenced $(2 \times 300)$ on an Illumina MiSeq platform (Illumina, San Diego, United States) as per the standard protocols of Majorbio Bio-Pharm Technology Co. Ltd. (Shanghai, China).

\section{Data Analysis}

\section{Comparison of Ramie Field Performance and Nutrient Concentration Between Genotypes}

Field performance and nutrient concentration among genotypes were compared using ANOVA (analysis of variance) in SAS 9.4 software (SAS Institute, Cary, NC, United States). Different plant genotypes and data collection times were considered independent variables. The mean of each trait was tested at the $p<0.05$ level using Duncan's multiple range test.

Evaluation of the overall field performance is a multi-criteria decision-making process that involves many factors. In this study, a membership function-derived normalized field performance index (NFPI) was used to comprehensively express overall field performance (Kang et al., 2012). The NFPI value of each field performance trait was calculated based on the following formula:

$$
\mathrm{y}_{i}(k)=\frac{x_{i}(k)-\min x(k)}{\max x(k)-\min x(k)}
$$

where $y_{i}(k)$ represents the NFPI value of the $i$ th genotype in terms of the $k$ th field performance trait, $x_{i}(k)$ denotes the field-recorded value of the $i$ th genotype in terms of the $k$ th field performance trait, and $\max x(k)$ and $\min x(k)$ represent the largest and smallest value of $x_{i}(k)$, respectively.

The overall NFPI of individual germplasm represents the average value of all calculated $y_{i}(k)$ values, as field performance indicators were assumed to make an equal contribution to the overall NFPI.

\section{Analysis of the Nutrient Changes Affecting the Poor Soil Tolerance Ability of Ramie}

Correlation analysis (hereafter referred to as CA analysis) was used to evaluate how nutrient cycling contributes to the poor soil tolerance ability of ramie. The poor soil tolerance ability of each germplasm was defined as its mean overall NFPI in the two 2018 growth periods. The independent variables of the CA analysis were the nutrient change indicators, and the dependent variable was the overall NFPI of the tested genotypes. The nutrient change indicators included nine from the first growth period (GP1: April-July) and nine from the second growth period (GP2: August to December). The first nine were the rates of change in the concentration of the three tested nutrients $(\mathrm{N}, \mathrm{P}$, and $\mathrm{K}$ ) in 
the three plant parts (leaves, stem, and root) over GP1 (i.e., July relative to April). The other nine were the rates of change in the concentration of these nutrients over GP2 (i.e., December relative to August). To reduce the dimensions of the complex indicators, principal component analysis (PCA) was carried out prior to the CA analysis to identify the most important variables relevant to nutrient change. Only indicators with significant $(p<0.05)$ correlations with principal components were used in the CA analysis. Based on the PCA results, 10 variables were selected, which were the rates of change in the concentration of $\mathrm{N}, \mathrm{P}$, and $\mathrm{K}$ in the root in GP2 (hereafter referred to as RN2, RP2, and RK2, respectively); rates of change in the concentration of $\mathrm{N}$ and $\mathrm{P}$ in the stem in GP1 (SN1 and SP1, respectively); rates of change in the concentration of $\mathrm{N}$ and $\mathrm{K}$ in the stem in GP2 (SN2 and SK2, respectively); rates of change in the concentration of $\mathrm{P}$ and $\mathrm{K}$ in the leaves in GP1 (LP1 and LK1, respectively); and the rate of change in the concentration of $\mathrm{K}$ in the root in GP1 (RK1). Correlations between the ramie's overall NFPI and the above 10 indicators were performed using the CORR procedure in SAS 9.4 software. Pearson's correlation coefficients and their significance were used to assess the strength of the correlations between NFPI and the 10 indicators.

\section{Microbial Composition Analysis}

The raw FASTQ sequencing reads were first demultiplexed, quality-filtered by Trimmomatic, and merged by FLASH following the criteria of Zhang et al. (2019). The structure of the soil microbial community was then analyzed in the free online Majorbio Platform ${ }^{2}$, which is based on the R packages USEARCH (Version 7.0), PICRUSt (Version 1.1.0), UNITE (Version 8.0), and FUNGuild (Version 1.0).

\section{RESULTS}

\section{Evaluation of the Poor Soil Tolerance of Different Ramie Genotypes Based on Their Field Performance}

The long-term plant field performance under poor soil conditions was evaluated assuming that a higher field performance corresponded to a stronger ability to tolerate poor soil conditions. In the ECS trial, the genotype $(\mathrm{G})$, plantation year $(\mathrm{Y})$, and growth period (GP) had a significant effect on all of the morphological traits measured (Table 2). Plants in November 2018 (eighth plantation year) were on average $23.1 \%$ lower in height ( 69.5 vs. $90.4 \mathrm{~cm}, p<0.001$ for the effect of Y), had 4.1 times more tillers ( 71.5 vs. $14, p<0.001$ ), and had $53.1 \%$ higher aboveground yield (179.0 vs. $\left.116.9 \mathrm{~g} / \mathrm{m}^{2}, p<0.001\right)$ compared with plants in November 2010 (the establishment year). Differences in the tested traits (Table 2) between the first and second growth periods were also significant $(p<0.01)$; specifically, plants at the end of GP1 were generally superior in terms of morphology compared with GP2 plants, with the exception of belowground yield. The belowground yield of the six genotypes was $2.08 \mathrm{~kg} / \mathrm{m}^{2}$ on average at the end of GP2, which

${ }^{2}$ www.majorbio.com

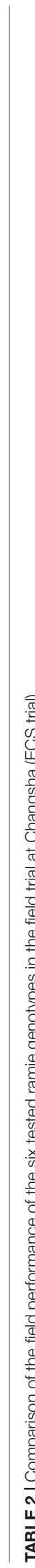

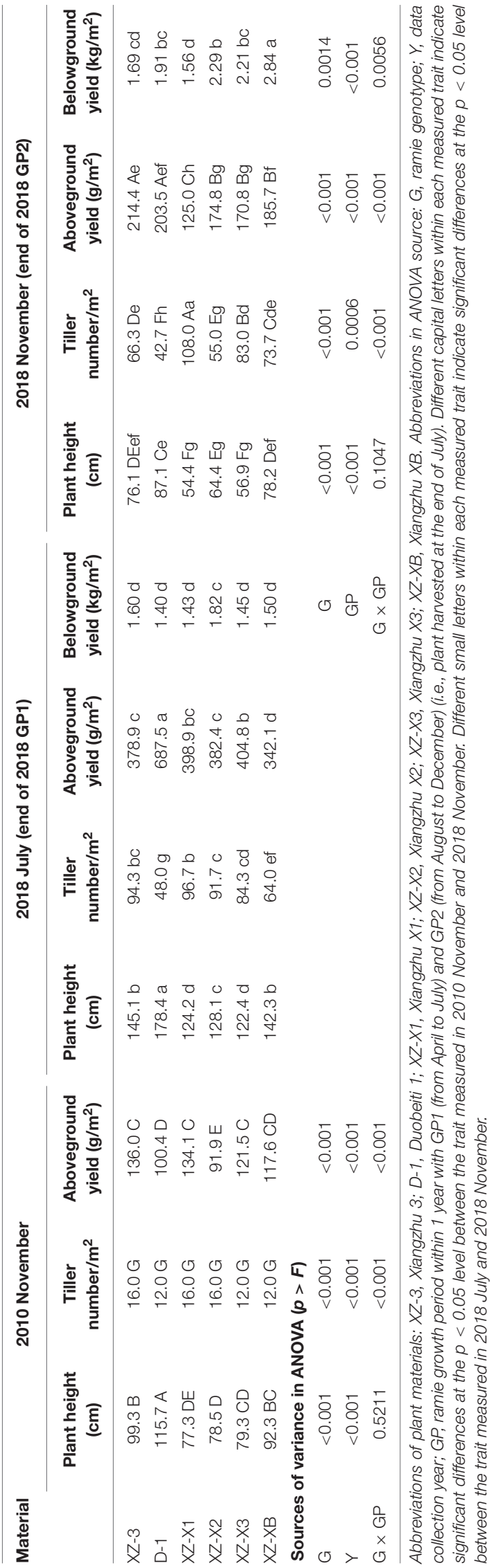


was $35.9 \%$ higher than that $\left(1.53 \mathrm{~kg} / \mathrm{m}^{2}\right)$ at the end of GP1. There were significant differences ( $p<0.001$ for the effect of $G$ ) in all morphological traits across the six tested genotypes. However, the ability to tolerate poor soil conditions (i.e., the overall NFPI) was not consistent over the years and also differed between growth periods (Table 3). At the end of 2010, XZ-3 had the highest overall NFPI (0.786), whereas XZ-X2 had the lowest overall NFPI (0.016). The other four genotypes showed a similar performance. However, in 2018, D-1 had the highest overall NFPI (0.667) at the end of GP1, followed by XZ-X2 (0.406); at the end of GP2, D-1 had a lower NFPI (0.716) than XZ-XB (0.799). A synthetic NFPI was used to comprehensively evaluate field performance, which was defined as the average value of the overall NFPI in GP1 and GP2. The genotypes from high performance (i.e., a better ability to thrive under poor soil conditions) to low performance were D-1, XZ-XB, XZ-3, XZ-X2, XZ-X3, and XZ-X1.

Although genotype and growth period still had a significant $(p<0.001)$ effect on ramie's field performance in the EHR trail, their effects were not fully consistent with those observed in the ECS trial (Table 4). The plants harvested at the end of GP2 were significantly $(p<0.001)$ taller than the plants at the end of GP1 (182.0 vs. $148.1 \mathrm{~cm})$ but had fewer tillers (28.5 vs. $37.8, p=0.003$ ), which is in contrast to the effect of growth period in the ECS trial. Additionally, the aboveground yield did not significantly differ between the two growth periods $(p=0.458)$. The comprehensive performance evaluation revealed that different genotypes performed similarly across the two growth periods, and the genotypes from high performance to low performance were XZ-XB, ZZ-1, XZ-X2, and XZ-3. This pattern is generally consistent with that in the ECS trial, in which $\mathrm{XZ}-\mathrm{XB}$ was the best-performing genotype (synthetic NFPI $=0.997$ ), and XZ-3 was the worst-performing genotype (synthetic NFPI $=0.000$ ).

\section{Relationship Between Seasonal Nutrient Cycling and Ramie Field Performance}

A similar pattern of change throughout the growing season was observed for TN, total phosphorus (TP), and total potassium (TK) (Figures 1-3). The nutrient concentration in the leaves and stems increased over time during GP1 (from April to July) but decreased during GP2 (from August to December). For example, at the end of GP1 (July), the average TN concentration was 19.2\% higher in the leaves (Figure 1A) $(23.0 \mathrm{~g} / \mathrm{kg})$ compared with the beginning of May $(19.3 \mathrm{~g} / \mathrm{kg})$ but significantly decreased from $18.1 \mathrm{~g} / \mathrm{kg}$ at the beginning of GP2 (September) to $16.2 \mathrm{~g} / \mathrm{kg}$ at the end of GP2 (December) $(p<0.001)$. Nutrient concentration in the root was relatively stable during GP1 but significantly increased over time in GP2 (Figures 1C, 2C, 3C). From May to July, the TK concentration in the root did not differ significantly $(p=0.1769)$. The concentration of all three tested macronutrients in the root significantly increased from September to December by $89.8 \%(\mathrm{TN}), 29.8 \%(\mathrm{TP})$, and $33.6 \%$ (TK).

Patterns of nutrient dynamics were complex and varied between genotypes (Figures 1-3). However, there was a general pattern in which genotypes that had a stronger tolerance of poor soil showed gradual increases in the $\mathrm{P}$ concentration of the leaves and $\mathrm{N}$ concentration of the stems during GP1 but rapid increases in the $\mathrm{N}, \mathrm{P}$, and $\mathrm{K}$ concentration of the root during GP2. In July 2018, the poorly performing genotype XZ-X3 had significantly $(p=0.0043)$ higher TP concentration in the leaves $(2.82 \mathrm{~g} / \mathrm{kg})$ compared with the other genotypes, as well as the highest LP1 (21.0\%). The best-performing genotype D-1 had a low LP1 (4.0\%). Similar changes were also observed for TN in the stem, as the lowest $\mathrm{SN} 1$ (5\%) was recorded for D-1, followed by XZ-3 (8.0\%) and XZ-XB (20.0\%); the worst-performing genotype XZ-X1 had a high SN1 (84.0\%). During GP2, the highest RN2 (163.0\%) was recorded for D-1, whereas the lowest RN2 (45.0\%) was recorded for XZ-X1. The highest RP2 (46.0\%) and RK2 $(54.0 \%)$ in the root were also recorded for D-1, whereas the lowest RP2 (6.0\%) was recorded for XZ-X1, and the lowest RK2 (14.0\%) was recorded for XZ-X3.

Nutrient concentration dynamics and synthetic NFPI in 2018 were analyzed by Pearson's correlation to determine the contribution of nutrient dynamics to improving the tolerance of ramie to poor soil conditions. The correlation results are shown in Table 5. Synthetic NFPI was significantly positively correlated with RN2 $(r=0.946, p=0.004)$ and RP2 $(r=0.932, p=0.007)$ but significantly negatively correlated with $\mathrm{SN1}(r=-0.877$, $p=0.022)$. The positive correlation between RK2 and synthetic NFPI was marginally significant $(r=0.795, p=0.059)$. The negative correlations of LP1 and SK2 with synthetic NFPI were

TABLE 3 | The normalized field performance index (NFPI) and order of the six ramie germplasms tested in the field trial at Changsha (ECS trial).

\begin{tabular}{|c|c|c|c|c|c|c|c|c|}
\hline \multirow[t]{2}{*}{ Material } & \multicolumn{2}{|c|}{2010 November } & \multicolumn{2}{|c|}{2018 July (end of 2018 GP1) } & \multicolumn{2}{|c|}{2018 November (end of 2018 GP2) } & \multicolumn{2}{|c|}{2018 average } \\
\hline & NFPI & Order & NFPI & Order & NFPI & Order & NFPI & Order \\
\hline$X Z-3$ & 0.786 & 1 & 0.331 & 3 & 0.589 & 3 & 0.460 & 3 \\
\hline D-1 & 0.596 & 2 & 0.667 & 1 & 0.716 & 2 & 0.691 & 1 \\
\hline$X Z-X 1$ & 0.478 & 4 & 0.091 & 6 & 0.000 & 6 & 0.045 & 6 \\
\hline$X Z-X 2$ & 0.016 & 6 & 0.406 & 2 & 0.477 & 4 & 0.441 & 4 \\
\hline$X Z-X 3$ & 0.362 & 5 & 0.101 & 5 & 0.366 & 5 & 0.233 & 5 \\
\hline$X Z-X B$ & 0.487 & 3 & 0.201 & 4 & 0.799 & 1 & 0.500 & 2 \\
\hline
\end{tabular}

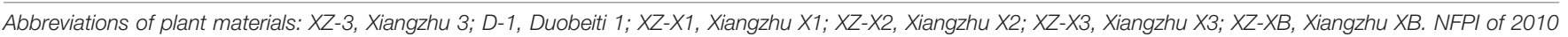



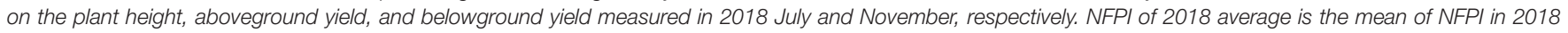
July and 2018 November. 

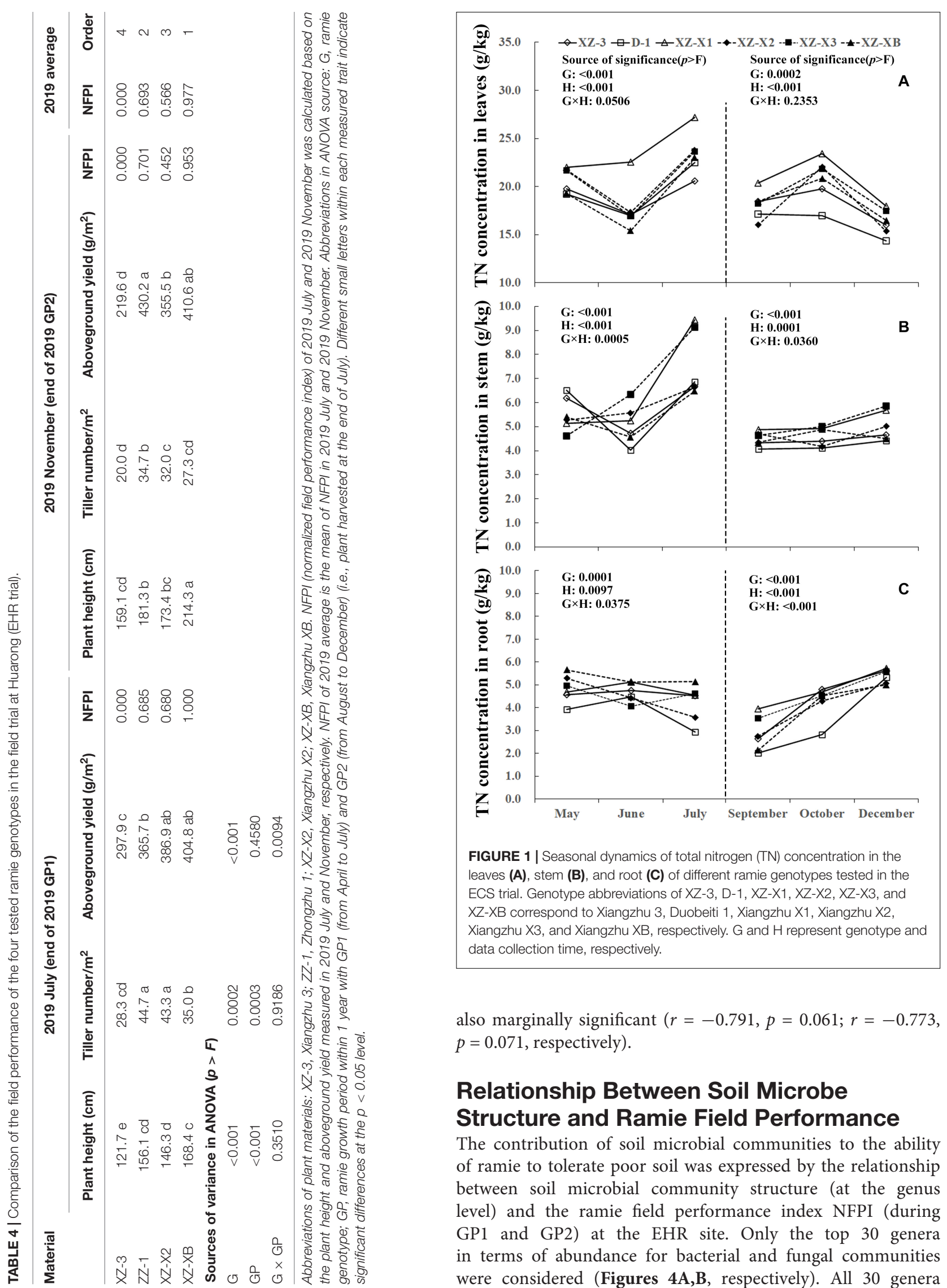

also marginally significant $(r=-0.791, p=0.061 ; r=-0.773$, $p=0.071$, respectively).

\section{Relationship Between Soil Microbe Structure and Ramie Field Performance}

The contribution of soil microbial communities to the ability of ramie to tolerate poor soil was expressed by the relationship between soil microbial community structure (at the genus level) and the ramie field performance index NFPI (during GP1 and GP2) at the EHR site. Only the top 30 genera in terms of abundance for bacterial and fungal communities were considered (Figures 4A,B, respectively). All 30 genera 


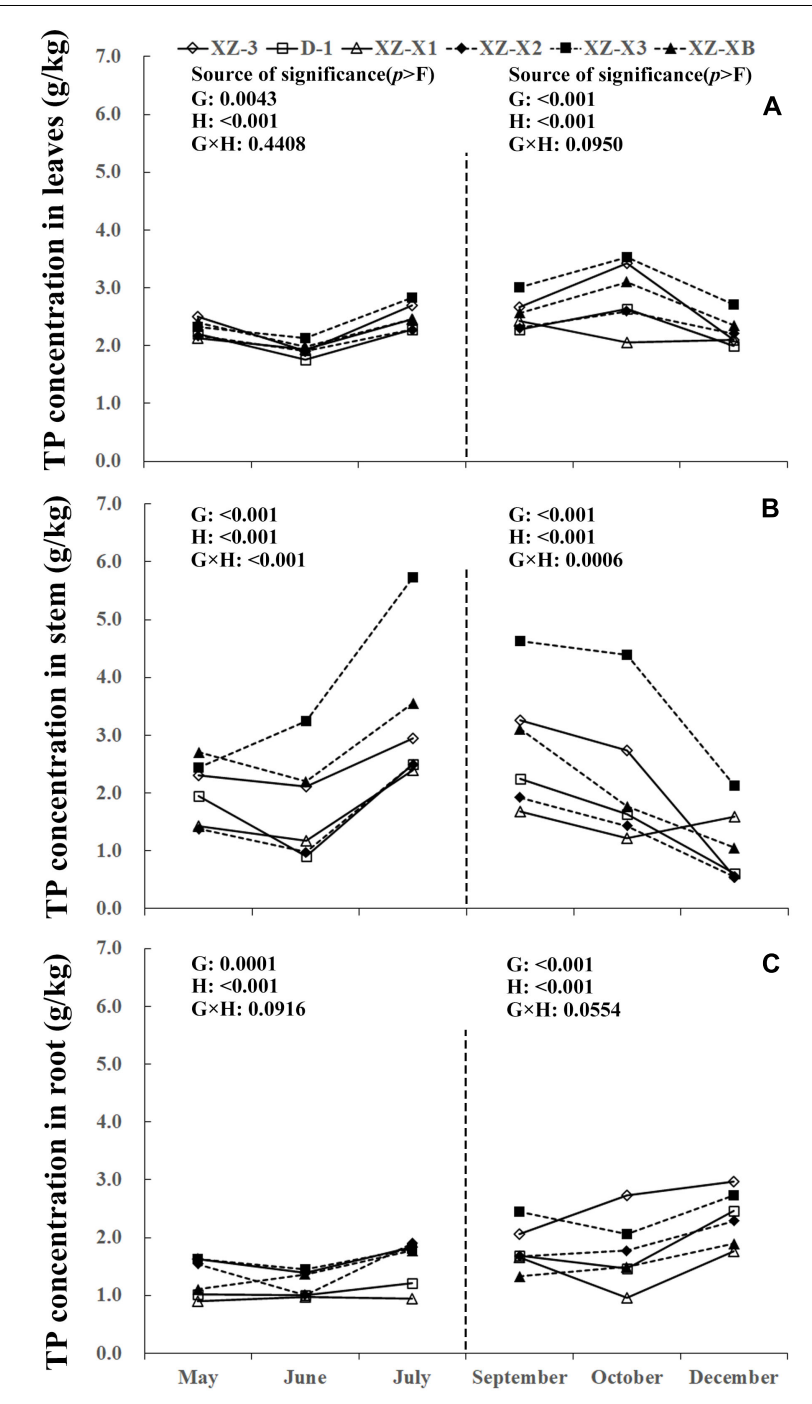

FIGURE 2 | Seasonal dynamics of total phosphorus (TP) concentration in the leaves (A), stem (B), and root (C) of different ramie genotypes tested in the ECS trial. Genotype abbreviations of XZ-3, D-1, XZ-X1, XZ-X2, XZ-X3, and XZ-XB correspond to Xiangzhu 3, Duobeiti 1, Xiangzhu X1, Xiangzhu X2, Xiangzhu $X 3$, and Xiangzhu $X B$, respectively. $G$ and $H$ represent genotype and data collection time, respectively.

of both bacterial and fungal communities were clustered into three groups: genera showing positive correlations with NFPI, genera showing negative correlations with NFPI, and genera unrelated to NFPI. There were six bacterial genera that were significantly positively correlated with ramie NFPI during GP1 (Pearson correlation coefficients in parentheses, $p<0.05$ ): IMCC26256 (Acidimicrobiia) (0.656), Candidatus_Solibacter (0.642), Bradyrhizobium (0.604), Gaiella (0.603), norank_f_SC-I84 (0.589), and norank_o_Gaiellales (0.581). During GP2, only norank_o_Gaiellales was significantly positively correlated with ramie field performance ( $p<0.05, r=0.582)$. Seven genera were negatively correlated with ramie field performance, but none of these correlations were significant (Figure 4A).

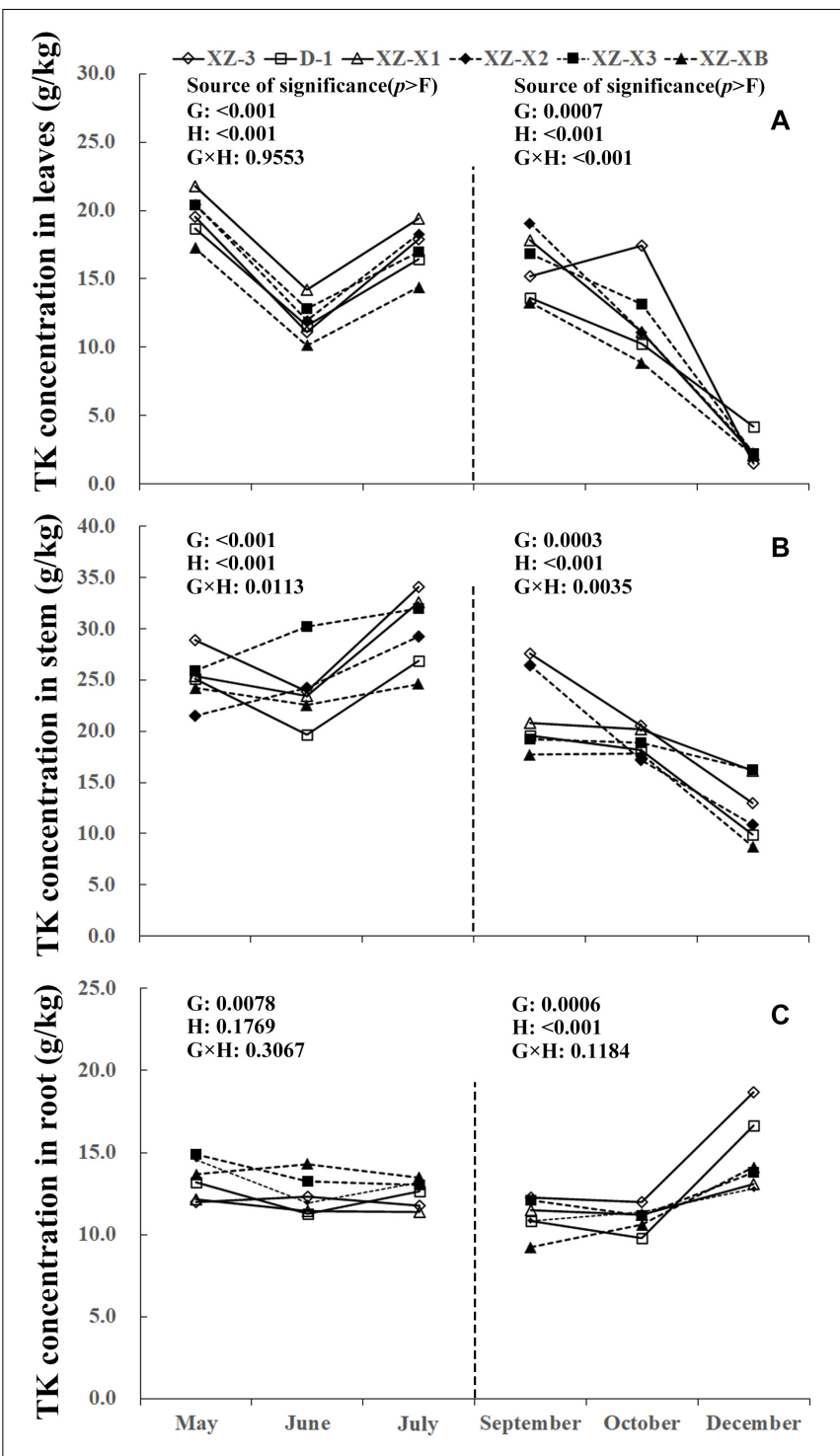

FIGURE 3 | Seasonal dynamics of total potassium (TK) concentration in the leaves $(\mathbf{A})$, stem $\mathbf{( B )}$, and root $\mathbf{( C )}$ of different ramie genotypes tested in the ECS trial. Genotype abbreviations of XZ-3, D-1, XZ-X1, XZ-X2, XZ-X3, and $X Z-X B$ represent Xiangzhu 3, Duobeiti 1, Xiangzhu X1, Xiangzhu X2, Xiangzhu $\mathrm{X} 3$, and Xiangzhu $\mathrm{XB}$, respectively. $\mathrm{G}$ and $\mathrm{H}$ represent genotype and data collection time, respectively.

Four fungal genera were significantly correlated with ramie field performance, including three negatively correlated genera and one positively correlated genus (Figure 4B). Cladosporium was the fungal genus that was the most strongly negatively correlated with NFPI during both GP1 $(r=-0.659, p<0.05)$ and GP2 $(r=-0.628, p<0.05)$. This genus was highly abundant $(20.3 \%)$ in the rhizosphere soil of the worst-performing genotype (XZ-3); by contrast, its abundance was only $1.33 \%$ in the rhizosphere soil of the best-performing genotype (XZ-XB). The genus Cladorrhinum had the lowest correlation coefficient $(r=-0.72, p<0.05)$ with NFPI, but this was only for GP2. This is also similar to Aspergillus, which only showed a significant 
TABLE 5 | Correlations between the nutrient change indicator and the ability of ramie to tolerate poor soil.

\begin{tabular}{|c|c|c|c|c|c|c|c|c|c|c|}
\hline & \multicolumn{10}{|c|}{ Nutrient change indicators } \\
\hline & RN2 & SN1 & SN2 & RP2 & SP1 & LP1 & RK1 & RK2 & SK2 & LK1 \\
\hline$r$ & 0.946 & -0.877 & -0.679 & 0.932 & -0.597 & -0.791 & 0.455 & 0.795 & -0.773 & 0.042 \\
\hline$p$ & 0.004 & 0.022 & 0.138 & 0.007 & 0.211 & 0.061 & 0.364 & 0.059 & 0.071 & 0.934 \\
\hline
\end{tabular}

$r$, Pearson's coefficients; $p$, p-values of the Pearson's coefficients. The first letters of the nutrient change indicators $R$, $S$, and $L$ represent the nutrient concentration in



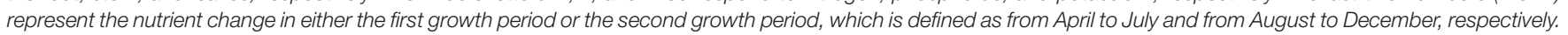

A

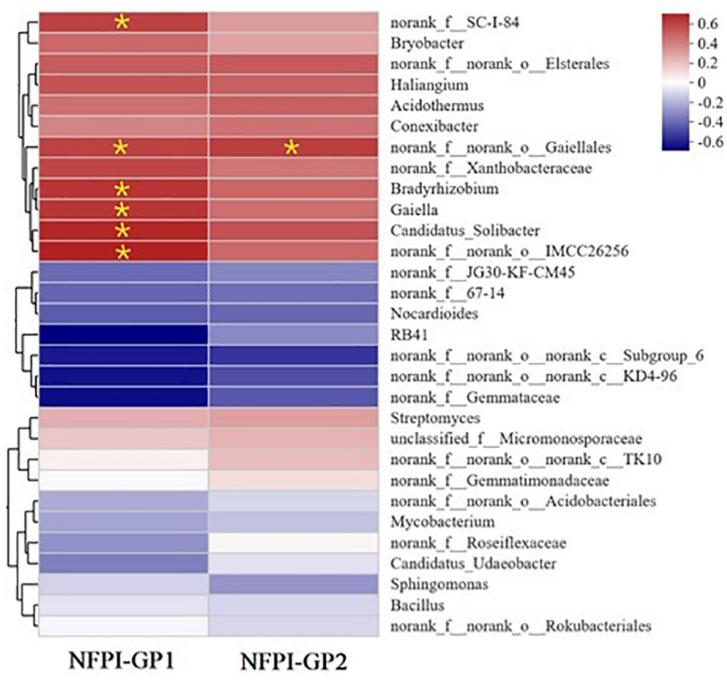

B

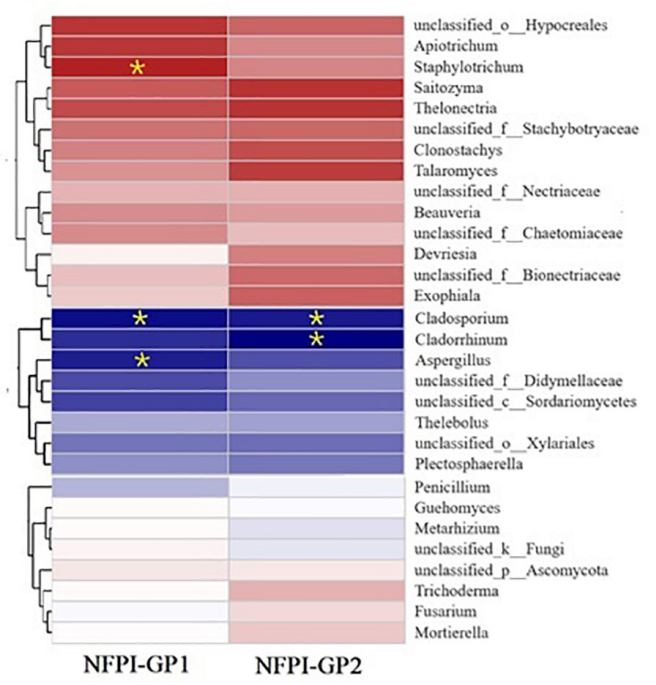

FIGURE 4 | Correlations between the rhizosphere soil microbial community structure and the tolerance of ramie to poor soil conditions (expressed by the normalized field performance index NFPI) during GP1 (from April to July 2019) and GP2 (from August to December 2019) at Huarong experimental station (i.e., EHR trial). The structure of soil microbial communities is expressed at the genus level of bacteria (A) and fungi (B). Significantly correlated $(p<0.05)$ relationships are marked by *.

negative correlation with NFPI during GP1 $(r=-0.623$, $p<0.05)$. Only Staphylotrichum had a significant positive correlation with NFPI during GP1 $(r=0.628, p<0.05)$. Additionally, Beauveria was dominant in the soil of the bestperforming genotype XZ-XB (25.96\%) but was not detected in the soil of the other genotypes.

\section{DISCUSSION}

\section{Optimal Timing for Evaluating Ramie Field Performance}

The first aim of this study was to evaluate the ability of different ramie genotypes to tolerate poor soil based on their field performance. The results of the ECS trial revealed that differences in plant field performance among genotypes between the establishment year and the eighth year after planting were not consistent. The fact that the perennial plants were not fully developed when they were established might explain these differences. Perennial plants generally need a few years to reach a stable level of performance (Jones et al., 2016; Xu et al., 2017; Stolarski et al., 2018). For example, comparison of morphological traits between different Miscanthus (a rhizomatous perennial species) genotypes has been generally conducted using data collected after the third year of planting (Xiang et al., 2018; Zheng C. et al., 2019). This should also be the case for perennial ramie. Angelini and Tavarini (2013) showed that stable field performance of ramie is generally achieved between the third to the 13th year after planting. This indicates that morphological traits should be measured from the third to the 13th year after cultivation when comparing differences in the field performance of different ramie genotypes. The results of both trials revealed significant performance differences between harvest times. In the ECS trial, plants performed better at the end of GP1 than at the end of GP2, which is consistent with the results of a previous study (Angelini and Tavarini, 2013). However, the opposite pattern was observed in the EHR trial. These results indicate that the plant phenotype throughout the entire growth period (divided by the mid-season harvest) within a year should be considered to comprehensively evaluate ramie performance. However, if more than two mid-season harvests are conducted, the contribution of each growth period should be weighed separately without taking the average given that ramie generally shows higher performance in early harvests compared with 
later harvests, especially under three to four mid-season harvest treatments (Liu et al., 2013; Wang et al., 2018).

\section{Effect of Seasonal Nutrient Cycling on the Ability of Ramie to Thrive Under Nutrient-Deficient Conditions}

As expected, $\mathrm{N}, \mathrm{P}$, and $\mathrm{K}$ retranslocation to the root at the end of the growing season helped ramie plants adapt to poor soil conditions. Our results also support the idea that nutrient retranslocation to the root is a survival strategy for perennial rhizomatous plants to adapt to nutrient-deficient conditions (Fife et al., 2008; Moreira and Fageria, 2009; Erley et al., 2010). However, delayed harvest is necessary for this strategy to be effective given that it provides sufficient time for the plant to complete the retranslocation process before harvest. However, this harvest regime is only recommended for cultivating ramie for ecological purposes, such as phytoremediation. This is mainly because delayed harvest damages ramie's fiber quality (Liu et al., 2013). Additionally, SN1 and LP1 were found to be negatively correlated with the ability of ramie to thrive under poor soil conditions. This may be the strategy by which ramie copes with the mid-season harvest. A lower SN1 and LP1 could reduce the amount of nutrients removed by the mid-season harvest. Overall, $\mathrm{N}$ translocation is more important than $\mathrm{P}$ translocation, followed by $\mathrm{K}$ translocation. This is consistent with a previous work showing that ramie growth is sensitive to $\mathrm{N}$ and $\mathrm{P}$ deficiency but not to $\mathrm{K}$ deficiency (Liu et al., 2012).

\section{Effect of Soil Microbial Structure on the Ability of Ramie to Thrive Under Nutrient-Deficient Conditions}

An additional aim of this study was to clarify the role of the soil microbial community on the ability of ramie to tolerate poor soil. The enrichment of the beneficial bacterial communities aided in the adaptation of ramie to poor soil conditions. Of the six significantly enriched bacterial genera, most have been shown to facilitate plant growth. For example, Bradyrhizobium are $\mathrm{N}$-fixing bacteria that can minimize the effects of nitrogen deficiency on plant growth (Hara et al., 2019; Ormeño-Orrillo and Martínez-Romero, 2019). Gaiella and norank_o_Gaiellales are both Actinobacteria, which can promote plant growth by increasing nutrient availability and assimilation (Franco-Correa and ChavarroAnzola, 2016; Novello et al., 2017; Severino et al., 2019). Candidatus_Solbacter can reduce moisture and nutrient fluctuations in stressful soil environments through the production of biofilms (Pearce et al., 2012). It can thus improve the ability of plants to withstand extreme environmental variation in moisture and nutrient availability (Challacombe et al., 2011). Although the exact functions of norank_f_SCI-84 and nornak_o_IMCC26256 remain unclear, this study confirms that they are closely tied to ramie growth under nutrient-deficient conditions. In contrast to the positive effect of bacterial communities, a reduction in harmful fungal communities is another strategy by which ramie can adapt to poor soil conditions. Of the significantly related fungal genera, Cladosporium can produce antimicrobial metabolites (Yehia et al., 2020) that can kill certain types of beneficial bacteria such as $\mathrm{N}$-fixing bacteria. Aspergillus can induce plant diseases that can reduce plant yields (Larbi-Koranteng et al., 2020). Cladorrhinum negatively affected ramie growth in this study, in contrast to its potential utility for biocontrol and its ability to promote plant growth (Carmarán et al., 2015). This may stem from the negative effect of some undetected species, but this hypothesis requires confirmation in future trials.

\section{CONCLUSION}

Based on two long-term (8-9 years) field trials of ramie cultivation, the genotypes of Duobeiti 1 and Xiangzhu XB were shown to be highly tolerant of poor soil. Low nutrient content in the stem and leaves and $\mathrm{N}$ and $\mathrm{P}$ retranslocation to the root at the end of the growing season are strategies underlying the ability of these genotypes to adapt to poor soil conditions. The enrichment of beneficial bacterial communities and the decrease in harmful fungal communities also help ramie plants adapt to poor soil conditions.

\section{DATA AVAILABILITY STATEMENT}

The datasets presented in this study can be found in online repositories. The names of the repository/repositories and accession number(s) can be found below: CNCB-NGDC (accession: CRA003958; https://bigd.big.ac.cn/gsa).

\section{AUTHOR CONTRIBUTIONS}

YJ and HX designed the experiments. SW performed the experiments and wrote the manuscript. SW and SX analyzed the data. YJ, SX, and YI reviewed and edited the manuscript. All authors read and approved the manuscript for submission.

\section{FUNDING}

This study was supported by grants from projects of the Foundation for the Construction of Innovative Hunan (2019RS1051), the National Natural Science Foundation of China (31872877), and China's National Key R\&D Program (2019YFD1002205).

\section{ACKNOWLEDGMENTS}

We thank all the people who extended their help in this study in the process of field sampling and chemical analysis. 


\section{REFERENCES}

Angelini, G., and Tavarini, S. (2013). Ramie [Boehmeria nivea (L.) Gaud.] as a potential new Fibre crop for the mediterranean region: growth, crop yield and fibre quality in a long-term field experiment in Central Italy. Ind. Crop. Prod. 51, 138-144. doi: 10.1016/j.indcrop.2013.09.009

Bao, S. D. (2000). Soil Agrochemical Analysis, 3rd Edn, Beijing: China Agricultural Press.

Carmarán, C. C., Berretta, M., Martínez, S., Barrera, V., Munaut, F., and Gasoni, L. (2015). Species diversity of Cladorrhinum in argentina and description of a new species, Cladorrhinum australe. Mycol. Prog. 14, 1-11. doi: 10.1007/s11557015-1106-3

Challacombe, J. F., Eichorst, S. A., Hauser, L., Land, M., Xie, G., and Kuske, C. R. (2011). Biological consequences of ancient gene acquisition and duplication in the large genome of Candidatus Solibater usitatus Ellin6076. PLoS One 6:e0024882. doi: 10.1371/journal.pone.0024882

Erley, G. S. A., Dewi, E. R., Nikus, O., and Horst, W. J. (2010). Genotypic differences in nitrogen efficiency of white cabbage (Brassica oleracea L.). Plant Soil 328, 313-325. doi: 10.1007/s11104-009-0111-1

Fife, D. N., Nambiar, E. K. S., and Saur, E. (2008). Retranslocation of foliar nutrients in evergreen tree species planted in a Mediterranean environment. Tree Physiol. 28, 187-196. doi: 10.1093/treephys/28.2.187

Franco-Correa, M., and Chavarro-Anzola, V. (2016). "Actinobacteria as plant growth-promoting rhizobacteria," in Actinobacteria-Basics and Biotechnological Applications, eds D. Dhanasekaran and Y. Jiang (London: Intech), 249-270. doi: $10.5772 / 61291$

Hara, S., Morikawa, T., Wasai, S., Kasahara, Y., Koshiba, T., Yamazaki, K., et al. (2019). Identification of nitrogen-fixing Bradyrhizobium associated with roots of field-grown sorghum by metagenome and proteome analyses. Front. Microbiol. 10:407. doi: 10.3389/fmicb.2019.00407

Jones, M. B., Zimmermann, J., and Clifton-Brown, J. (2016). "Long-term yields and soil carbon sequestration from Miscanthus: a review," in Perennial Biomass Crops for a Resource Constrained World, eds S. Barth, D. Murphy-Bokern, O. Kalinina, G. Taylor, and M. Jones (Cham: Springer), 43-49. doi: 10.1007/978-3319-44530-4_4

Kang, W., Jie, Y., and Xing, H. (2012). Identification and evaluation of poor-soilfertility tolerance in Ramie Germplasms. J Plant Gene Resour. 13, 1023-1030.

Lan, M. M., Liu, C., Liu, S. J., Qiu, R. L., and Tang, Y. T. (2020). Phytostabilization of $\mathrm{Cd}$ and $\mathrm{Pb}$ in highly polluted farmland soils using ramie and amendments. Int. J. Environ. Res. Public Health 17:1661. doi: 10.3390/ijerph17051661

Larbi-Koranteng, S., Awuah, R. T., and Kankam, F. (2020). Biological control of black pod disease of cocoa (Theobroma cacao L.) with Bacillus amyloliquefaciens, Aspergillus sp. and Penicillium sp. in vitro and in the field. J. Microbiol. Antimicrob. 12, 52-63. doi: 10.5897/JMA2020.0434

Liu, L. J., Chen, H. Q., Dai, X. B., Wang, H., and Peng, D. X. (2012). Effect of planting density and fertilizer application on fiber yield of ramie (Boehmeria nivea). J. Integr. Agric. 11, 1199-1206. doi: 10.1016/S2095-3119(12)60115-4

Liu, L. J., Lao, C. G., Zhang, N., Chen, H. Q., Deng, G., Zhu, C., et al. (2013). The effect of new continuous harvest technology of ramie (Boehmeria nivea L.Gaud.) on fiber yield and quality. Ind. Crop. Prod. 44, 677-683. doi: 10.1016/ j.indcrop.2012.09.003

Liu, Y., and Ludewig, U. (2019). Nitrogen-dependent bacterial community shifts in root, rhizome and Rhizosphere of nutrient-efficient Miscanthus $x$ giganteus from long-term field trials. GCB Bioenergy 11, 1334-1347. doi: 10.1111/gcbb. 12634

Lwin, C. S., Seo, B. H., Kim, H. U., Owens, G. O., and Kim, K. R. (2018). Application of soil amendments to contaminated soils for heavy metal immobilization and improved soil quality-a critical review. Soil Sci. Plant Nutr. 64, 156-167. doi: 10.1080/00380768.2018.1440938

Mensah, A. K. (2015). Role of revegetation in restoring fertility of degraded mined soils in Ghana: a review. Int. J. Biodivers. Conserv. 7, 57-80. doi: 10.5897/ IJBC2014.0775

Moreira, A., and Fageria, N. K. (2009). Yield, uptake, and retranslocation of nutrients in banana plants cultivated in upland soil of central Amazonian. J. Plant. Nutr. 32, 443-457. doi: 10.1080/01904160802660750

Nasso, N. N. D., Roncucci, N., and Bonari, E. (2013). Seasonal dynamics of aboveground and belowground biomass and nutrient accumulation and remobilization in giant reed (Arundo donax L.): a three-year study on marginal land. Bioenerg. Res. 6, 725-736. doi: 10.1007/s12155-012-9289-9

Nasso, N. N. D., Roncucci, N., Triana, F., Tozzini, C., and Bonari, E. (2011). Seasonal nutrient dynamics and biomass quality of giant reed (Arundo donax L.) and miscanthus (Miscanthus $x$ giganteus Greef et Deuter) as energy crops. Italy J. Agron. 6:e24. doi: 10.4081/ija.2011.e24

Neilson, S., and Rajakaruna, N. (2014). "Phytoremediation of agricultural soils: using plants to clean metal-contaminated arable land," in Phytoremediation: Management of Enviromental Contaminants, eds A. A. Ansari, S. S. Gill, G. R. Lanza, and L. Newman (Cham: Springer), 159-168. doi: 10.1007/978-3-31910395-2_11

Novello, G., Gamalero, E., Bona, E., Boatti, L., Mignone, F., Massa, N., et al. (2017). The Rhizosphere bacterial microbiota of Vitis vinifera cv. Pinot Noir in an integrated pest management vineyard. Front. Microbiol. 8:e1528. doi: $10.3389 /$ fmicb. 2017.01528

Ormeño-Orrillo, E., and Martínez-Romero, E. (2019). A genomotaxonomy view of the Bradyrhizobium genus. Front. Microbiol. 10:1334. doi: 10.3389/fmicb.2019. 01334

Pearce, D. A., Newsham, K. K., Thorne, M. A. S., Clavo-Bado, L., Kresk, M., Laskaris, P., et al. (2012). Metagenomic analysis of a southern maritime Antartic soil. Front. Microbiol. 3:403. doi: 10.3389/fmicb.2012.00403

Rehman, M., Gang, D., Liu, Q., Chen, Y., Wang, B., Peng, D., et al. (2019). Ramie, a multipurpose crop: potential applications, constraints and improvement strategies. Ind. Crop. Prod. 137, 300-307. doi: 10.1016/j.indcrop.2019. 05.029

Rosariastuti, R., Barokah, U., and Supriyadi, S. (2018). Phytoremediation of $\mathrm{Pb}$ contaminated paddy field using combination of Agrobacterium sp. I3, compost and ramie (Boehmeria nivea). J. Degrade Min. Land Manag. 5, 1381-1388. doi: $10.15243 / \mathrm{jdmlm} .2018 .054 .1381$

Ruf, T., Schmidt, A., Delfosse, P., and Emmerling, C. (2017). Harvest date of Miscanthus $\times$ Giganteus affects nutrient cycling, biomass development and soil quality. Biomass Bioenerg. 100, 62-73. doi: 10.1016/j.biombioe.2017.03.010

Satya, P., Mitra, S., and Ray, D. P. (2019). "Ramie (Boehmeria nivea L. Gaud) genetic improvement," in Advances in Plant Breeding Strategies: Industrial and Food Crops, eds J. M. Al-Khayri, S. M. Jain, and D. V. Johnson (Cham: Springer International Publishing), 115-150. doi: 10.1007/978-3-030-23265-8 5

Severino, R., Froufe, H. J. C., Barroso, C., Albuquerque, L., Lobo-da-Cunha, A., da Costa, M. S., et al. (2019). High-quality draft genome sequence of Gaiella occulta isolated from a $150 \mathrm{~m}$ deep mineral water borehole and comparison with the genome sequences of other deep-branching lineages of the phylum Actinobacteria. Microbiologyopen 8:e00840. doi: 10.1002/mbo3.840

Stolarski, M. J., Śnieg, M., Krzyżaniak, M., Tworkowski, J., and Szczukowski, S. (2018). Short rotation coppices, grasses and other herbaceous crops: productivity and yield energy value versus 26 genotypes. Biomass Bioenerg. 119 , 109-120. doi: 10.1016/j.biombioe.2018.09.014

Tatar, Ö, Ilker, E., Tonk, F. A., Aygün, H., and Çaylak, Ö (2010). Impact of different nitrogen and potassium application on yield and fiber quality of ramie (Boehmeria nivea). Int. J. Agric. Biol. 14, 369-372. doi: 10.1016/j.compag.2010. 02.001

Wang, Y. Z., Dai, Q. Z., Wang, M. S., Yu, C. M., Tang, S. W., and Xiong, H. P. (2018). The study of forage yield and quality of ramie among different cutting times. Plant Fiber Sci. China 40, 85-91.

Xiang, W., Xue, S., Qin, S., Xiao, L., Liu, F., and Yi, Z. (2018). Development of a multi-criteria decision making model for evaluating the energy potential of Miscanthus germplasms for bioenergy production. Ind. Crop. Prod. 125, 602-615. doi: 10.1016/j.indcrop.2018.09.050

Xu, J., Gauder, M., Gruber, S., and Claupein, W. (2017). Yields of annual and perennial energy crops in a 12-year field trial. Agron. J. 109, 811-821. doi: 10.2134/agronj2015.0501

Yehia, R. S., Osman, G. H., Assaggaf, H., Salem, R., and Mohamed, M. S. (2020). Isolation of potential antimicrobial metabolites from endophytic fungus Cladosporium cladosporioides from endemic plant Zygophyllum mandavillei. S. Afr. J. Bot. 134, 296-302. doi: 10.1016/j.sajb.2020. 02.033

Zeng, X. F., Cui, G. X., and Ouyang, X. R. (2013). The effects of fertilization and plant density on fiber yield and quality of ramie. Chin. Agric. Sci. Bull. 29, $146-150$. 
Zhang, M., Riaz, M., Zhang, L., El-desouki, Z., and Jiang, C. (2019). Biochar induces changes to basic soil properties and bacterial communities of different soils to varying degrees at $25 \mathrm{~mm}$ rainfall: more effective on acidic soils. Front. Microbiol. 10:1321. doi: 10.3389/fmicb.2019.01321

Zheng, J., You, S., and Yang, Y. (2019). Review on the remediation of heavy metal antimony contaminated soil. IOP Conf. Ser. Earth Environ. Sci. 242:052010. doi: 10.1088/1755-1315/242/5/052010

Zheng, C., Iqbal, Y., Labonte, N., Sun, G., Feng, H., Yi, Z., et al. (2019). Performance of switchgrass and Miscanthus genotypes on marginal land in the Yellow River Delta. Ind. Crop. Prod. 141:111773. doi: 10.1016/j.indcrop.2019.111773
Conflict of Interest: The authors declare that the research was conducted in the absence of any commercial or financial relationships that could be construed as a potential conflict of interest.

Copyright (c) $2021 \mathrm{Wu}$, Xue, Iqbal, Xing and Jie. This is an open-access article distributed under the terms of the Creative Commons Attribution License (CC BY). The use, distribution or reproduction in other forums is permitted, provided the original author(s) and the copyright owner(s) are credited and that the original publication in this journal is cited, in accordance with accepted academic practice. No use, distribution or reproduction is permitted which does not comply with these terms. 